\title{
Avaliação antropométrica e dietética de hipertensos atendidos em ambulatório de um hospital universitário
}

\author{
Anthropometric and dietetic evaluation of hypertensive \\ outpatients from a university hospital
}

Poliana Coelho CABRAL ${ }^{1}$

Ana Maria de Carvalho Albuquerque MELO'

Tânia Campos Fell AMADO'

Rijane Maria de Andrade Barros dos SANTOS

\section{RE S U M O}

O objetivo deste trabalho foi avaliar o estado nutricional e o consumo de nutrientes de hipertensos, na faixa etária de 18 a 74 anos, atendidos ambulatorialmente no Hospital das Clínicas da Universidade Federal de Pernambuco. A amostra, escolhida ao acaso, foi composta por 156 indivíduos de ambos os sexos (51 homens e 105 mulheres). As análises mostraram uma elevada prevalência de pacientes $(73,1 \%)$ com peso acima da média e entre eles a hipertensão se associou significativamente com a obesidade (Índice de Massa Corporal $\geq 30 \mathrm{Kg} / \mathrm{m}^{2}$ ) e com o sobrepeso (Índice de Massa Corporal $\geq 25 \mathrm{e}<30 \mathrm{Kg} / \mathrm{m}^{2}$ ) mas não com a distribuição central de gordura. Em relação ao consumo de nutrientes, os achados desta pesquisa revelaram um baixo consumo energético, o qual não pode explicar a alta prevalência de sobrepeso e obesidade. De uma forma geral, exceto pelo cálcio, a ingestão média diária de nutrientes esteve adequada. No entanto, o consumo diário de sódio foi alto, 270 e $260 \mathrm{mEq}$ para homens e mulheres, respectivamente.

Termos de indexação: hipertensão, estado nutricional, consumo de nutrientes, obesidade, antropometria.

\section{A B S T R A C T}

The purpose of this research was to evaluate the nutritional status and the nutrients intake of hypertensive outpatients, aged 18 to 74 years old, from the Hospital das Clínicas - Universidade Federal de Pernambuco, Brazil. The sample, chosen at random, consisted of 156 individuals of both sexes (51 males and 105 females). The analyses showed a high prevalence of patients (73.1\%) with weight above average and among them the hypertension was significantly associated with obesity (Body Mass Index $\geq 30 \mathrm{Kg} / \mathrm{h}^{2}$ ) and overweight (Body Mass Index $\geq 25$ and $<30 \mathrm{Kg} / \mathrm{h}^{2}$ ) but not with centrally deposited body fat. Regarding the nutrients intake, the

\footnotetext{
1 Laboratório de Nutrição Clínica, Departamento de Nutrição, Universidade Federal de Pernambuco. Campus Universitário, 50670-901, Recife, PE, Brasil. Correspondência para/Correspondence to: P.C. CABRAL.E-mail: pccabral@uol.com.br
} 
findings of this survey revealed a low caloric consumption that can not explain the high prevalence of overweight and obesity. In general, the average daily intake of nutrients was adequate, except for calcium. However, the daily consumption of sodium was very high, 270 and $260 \mathrm{mEq}$ for men and women, respectively.

Index terms: hypertension, nutritional status, nutrients consumption, obesity, anthropometry.

\section{N T R O D U Ç Ã O}

A hipertensão arterial é um dos maiores problemas de saúde no Brasil, estando associada a sérios riscos de morbimortalidade cardiovascular, contribuindo diretamente para a ocorrência de infarto agudo do miocárdio, acidente vascular cerebral, insuficiência cardíaca congestiva, insuficiência arterial periférica e morte prematura (Silveira et al., 2001).

Com relação aos dados de prevalência, apesar da grande diversidade metodológica encontrada na maioria dos estudos epidemiológicos brasileiros (Lessa, 1998), o que dificulta a identificação da realidade nacional, estima-se que $15 \%$ a $20 \%$ da população adulta brasileira seja hipertensa (Silveira et al., 2001).

Até recentemente, a hipertensão era diagnosticada e categorizada principalmente com base na pressão diastólica. No entanto, hoje se sabe que a morbimortalidade aumenta quando se eleva tanto esta quanto a sistólica (Massie, 1997). Dessa forma, considera-se atualmente hipertensão uma Pressão Arterial (PA) persistentemente alta, definida como pressão sanguínea sistólica $\geq 140 \mathrm{mmHg}$ e/ou pressão sanguínea diastólica $\geq 90 \mathrm{mmHg}$ (Sixth Report..., 1997).

Uma das classificações mais aceitas, para a PA, é a do Joint National Committee (Sixth Report..., 1997), a qual também leva em consideração níveis altos "normais" (valores de 130 a 139 e 85 a $89 \mathrm{mmHg}$ ), procedimento de grande interesse em se tratando de prevenção primária, ou seja, ação antes da ocorrência da doença.

Partindo desta perspectiva, torna-se indispensável o conhecimento dos fatores de risco associados à hipertensão.
Em relação aos fatores de risco - idade, hereditariedade, raça e sexo, pouco ou nada pode ser feito, pois a hipertensão tem um forte componente familiar e afeta mais intensamente os homens (situação invertida após a menopausa, quando as mulheres apresentam as maiores prevalências), negros e idosos (Achutti \& Achutti, 1997). Quanto aos fatores para os quais existe possibilidade de intervenção, um dos mais importantes é a obesidade, apesar de os mecanismos fisiopatológicos envolvidos nessa relação ainda permanecerem obscuros (Bertolami, 1994).

No que se refere aos fatores dietéticos poucos assuntos têm sido tão debatidos quanto a relação dieta versus hipertensão. De fato, a possível associação entre sal $(\mathrm{NaCl})$ e a PA tem gerado muita discussão com resultados ainda controversos (Campese \& Bianchi, 1997); além disso, em investigações acerca de outros elementos, já existem evidências consideráveis, porém inconclusivas, sobre o papel do potássio, cálcio, magnésio, cafeína, gorduras, proteínas, álcool, fumo, fibras, e outras substâncias (Adrogué \& Wesson, 1996; Massie, 1997; Krummel, 1998).

Entretanto, como os mecanismos da hipertensão ainda não foram completamente esclarecidos, torna-se difícil encontrar uma resposta simples sobre a importância dos fatores dietéticos na sua etiologia, e é mais difícil ainda saber como deve ser a dieta dos indivíduos hipertensos. Desse modo, levando-se em consideração a elevada prevalência da hipertensão no Brasil, bem como a insuficiência de dados que possam seguramente definir uma correta orientação nutricional, a finalidade principal deste trabalho foi, em primeiro lugar, verificar a possível associação entre estado nutricional e a ocorrência de hipertensão e, em 
segundo, descrever o consumo alimentar, na fase anterior ao tratamento, visando conhecer, em um grupo de hipertensos em particular, o perfil de ingestão dos nutrientes mais amplamente citados na literatura como envolvidos na gênese da hipertensão arterial.

\section{CASUÍSTICAE MÉTODOS}

Para esta investigação de caráter transversal, foi utilizada amostra de indivíduos com hipertensão essencial, na faixa etária de 18 a 74 anos, encaminhados para orientação dietoterápica no Ambulatório Geral de Nutrição do Hospital das Clínicas (HC) da Universidade Federal de Pernambuco (UFPE).

O Serviço de Nutrição do HC/UFPE vem atendendo na área ambulatorial desde 1960 e conta hoje com nove ambulatórios especializados e um para atendimento geral e triagem. Neste último, segundo levantamento realizado por Burgos et al. (1998), a média anual de primeiro atendimento foi de 1500 pacientes, com uma prevalência de hipertensão essencial em torno de $22 \%$. Para o cálculo do tamanho amostral, adotou-se essa cifra (22\%) como a prevalência estimada de hipertensão, com uma margem de erro de 7\% e um nível de significância de 5\% para rejeição da hipótese de nulidade, resultando em um número de pacientes igual a 123 . Entretanto, com o objetivo de corrigir eventuais perdas durante o processo de coleta dos dados, decidiu-se elevar a amostra para 156 pacientes.

Algumas considerações necessitam ser feitas sobre o processo de amostragem. Como se trata de um levantamento visando a identificação dos hábitos alimentares em uma fase anterior ao tratamento, só foram selecionados os indivíduos que não haviam, até o momento da consulta, modificado seu consumo em virtude da doença. No entanto, tendo em vista a dificuldade de se encontrar pacientes nessas condições, qualquer hipertenso adulto encaminhado ao ambulatório, caso preenchesse os critérios de inclusão, era convidado a fazer parte do estudo, procedimento adotado até a aquisição do número de pacientes estipulado pelo cálculo amostral.

A entrevista era composta por uma fase inicial de coleta de dados sociodemográficos e clínicos, tais como sexo, idade, ocupação, grau de instrução, renda familiar e nível de pressão arterial, com o objetivo de melhor conhecer a clientela sob estudo.

Para avaliar o estado nutricional do grupo em estudo foi utilizado o Índice de Massa Corporal (IMC). Esse indicador vem sendo largamente empregado porque é simples, correlaciona-se com outras medidas corporais, não necessita de padrão de referência e tem sido reconhecido como o indicador que isoladamente permite o melhor diagnóstico da situação nutricional de adultos em nível coletivo (Cabral, 1994). A classificação utilizada foi a recomendada pela Organização Mundial da Saúde (OMS) (World Health..., 1995).

Em caráter complementar, com o objetivo de identificar o padrão de distribuição da massa adiposa, a qual, segundo vários estudos, tem maior valor preditivo de morbimortalidade que a quantidade total de gordura (Rimm et al., 1995; Lerário et al., 1997; Gillum et al., 1998; Rexrode et al., 1998), foi utilizada a Relação Cintura/Quadril (RCQ). Esta medida antropométrica representa a relação entre a circunferência da cintura (no menor diâmetro do abdome) e a circunferência do quadril (na altura das cristas ilíacas) (Gus et al., 1998). Um resultado superior a 1,0 e 0,85 em homens e mulheres, respectivamente, é considerado como fator de risco cardiovascular mais importante do que um IMC > 30,0kg/m² (Williams et al., 1987) e, portanto, esses pontos de corte foram considerados neste trabalho.

Os instrumentos utilizados na coleta dos parâmetros antropométricos citados acima foram: balança da marca Filizola com capacidade para 150kg; antropômetro da balança Filizola com limite para 1,90m; fita métrica de fibra de vidro, em centímetros, com precisão de milímetros.

Para o registro da ingestão de alimentos o método escolhido foi o Recordatório de 24 horas 
(aplicado apenas no dia da consulta). Cada indivíduo foi entrevistado por um nutricionista sobre tudo o que tinha ingerido no almoço, jantar e lanches do dia anterior e no desjejum do dia da entrevista. A realização do inquérito em apenas um dia foi decorrente da dificuldade operacional de se ter acesso mais vezes ao mesmo paciente.

Com o objetivo de tentar identificar, de forma grosseira, a ingestão do sal adicionado ao alimento, ou pronto (na mesa) ou no momento do preparo da refeição, questionou-se o paciente sobre o consumo médio mensal da família, do qual foi retirado um per capita.

Os resultados obtidos (exceto para fibras e colesterol) foram comparados às Recomendações do National Research Council (National Research..., 1989), adotando-se como padrão para ingestão de fibras o valor de 20 a 30g/dia (Mahan \& Arlin, 1995) utilizando-se para o colesterol dietético a recomendação proposta pelo National Cholesterol Education Program (National Cholesterol..., 1993), que é de no máximo 300mg por dia.

Os dados desta pesquisa foram processados e analisados utilizando-se o software Epi Info (World Health..., 1997).

No estudo comparativo entre excesso de peso e hipertensão arterial foram aplicados testes não paramétricos (Qui-quadrado convencional com correção de Yates e teste de Probabilidades de Fisher). Ressalta-se que este último foi empregado quando os resultados não preenchiam os requisitos para aplicação do Qui-quadrado. Em caráter complementar, para os resultados de natureza paramétrica Média ( $\bar{X}$ ) e Desvio-padrão (DP), a Análise de Variância (ANOVA) foi utilizada com a

Tabela 1. Características Sociodemográficas e Clínicas dos Hipertensos atendidos em $1^{\text {a }}$ consulta no Ambulatório Geral de Nutrição do HC/UFPE, Recife, 1999.

\begin{tabular}{|c|c|c|c|}
\hline & & $n$ & $\%$ \\
\hline \multirow[t]{2}{*}{ Sexo } & Homens & 51 & 32,7 \\
\hline & Mulheres & 105 & 67,3 \\
\hline \multirow[t]{4}{*}{ Idade (anos) } & $18-34$ & 18 & 11,5 \\
\hline & $35-44$ & 37 & 23,7 \\
\hline & $45-54$ & 40 & 25,7 \\
\hline & $\geq 55$ & 61 & 39,1 \\
\hline \multirow[t]{3}{*}{ Nível de Atividade } & Leve & 121 & 77,6 \\
\hline & Moderada & 33 & 21,1 \\
\hline & Intensa & 2 & 1,3 \\
\hline \multirow[t]{3}{*}{ Grau de Instrução } & Não Alfabetizado & 58 & 37,2 \\
\hline & $1^{\circ} \mathrm{Grau} \mathrm{C/I^{* }}$ & 85 & 54,5 \\
\hline & $2^{\circ} \mathrm{Grau} \mathrm{C/I^{* }}$ & 13 & 8,3 \\
\hline Renda Domiciliar & $<2$ & 68 & 43,6 \\
\hline \multirow[t]{2}{*}{ (Salário Mínimo) } & $\geq 2$ e $<4$ & 63 & 40,4 \\
\hline & $\geq 4$ & 25 & 16,0 \\
\hline \multirow[t]{4}{*}{ Pressão Arterial (PA) } & Normal (controlada) & 36 & 23,1 \\
\hline & • HA leve & 41 & 26,3 \\
\hline & - HA moderada & 62 & 39,7 \\
\hline & - HA severa + muito severa & 17 & 10,9 \\
\hline
\end{tabular}

Amostra $\mathrm{n}=156 ;{ }^{*}$ () $\mathrm{C} / \mathrm{I}=$ Completo ou Incompleto; $(\bullet) \mathrm{HA}=$ Hipertensão Arterial. 
finalidade de detectar a ocorrência ou não de diferenças estatísticas significativas entre os valores comparados.

Em uma etapa anterior, a análise da composição da dieta foi realizada através do software de apoio à Nutrição da Escola Paulista de Medicina (Programa..., 1993), o qual utiliza como base para cálculo a Tabela de Composição de Alimentos Americana, denominada Composition of foods-raw, processed, prepared agriculture handbook.

\section{RESULTADOSE DISCUSSÃO}

A amostra ( $n=156)$ é constituída basicamente por indivíduos acima dos 45 anos, com uma média de idade em torno de 49,8 \pm 15,6 anos (Tabela 1). Este resultado está de acordo com relatos de diversos autores, os quais evidenciaram elevação da incidência de hipertensão com o aumento da idade (Almeida, 1996; Achutti \& Achutti, 1997).

Em relação à distribuição por gênero, esta pesquisa constatou um predomínio bastante expressivo do sexo feminino, chegando a $67,3 \%$ da amostra. Esta constatação não é nenhuma surpresa, tendo em vista a faixa etária média desses pacientes e as evidências relatadas na literatura de que a hipertensão é mais prevalente no sexo masculino até aproximadamente os 45 a 50 anos, quando então essa situação se inverte (Lessa, 1998). Outra possível explicação é a maior preocupação da mulher com a sua saúde, levando-a a procurar com mais freqüência o atendimento médico, fato já observado por alguns autores (Pinotti, 1987; Veras et al., 1987; Must et al., 1992).

Destaca-se que $37,2 \%$ dos entrevistados eram analfabetos e mais de $80,0 \%$ subsistiam com uma renda domiciliar inferior a quatro salários mínimos, correspondendo, na época da coleta dos dados, a cerca de $R \$ 550,00$ a renda famíliar. Estas condições certamente interferem na compreensão da doença e na adesão ao tratamento proposto.

Quanto às características clínicas da amostra estudada, de acordo com os pontos de corte recomendados pelo Joint National Committee (Sixth Report..., 1997), 66,0\% dos pacientes apresentavam Hipertensão Arterial (HA) de leve a moderada, enquanto $10,9 \%$ foram classificados como hipertensos severos ou muito severos.

Tabela 2. Distribuição dos indivíduos com hipertensão leve, moderada e severa segundo os pontos de corte de IMC e RCQ•, Recife, 1999.

\begin{tabular}{|c|c|c|c|c|c|c|c|c|}
\hline \multirow{3}{*}{ IMC $\left(\mathrm{kg} / \mathrm{m}^{2}\right)$} & \multicolumn{6}{|c|}{ Níveis de Pressão Arterial (PA) } & & \\
\hline & \multicolumn{2}{|c|}{ *Nível A } & \multicolumn{2}{|c|}{ *Nível B } & \multicolumn{2}{|c|}{ *Nível C } & \multicolumn{2}{|c|}{ Total } \\
\hline & $n$ & $\%$ & $n$ & $\%$ & $n$ & $\%$ & $n$ & $\%$ \\
\hline 18,5 a 24,9 & 21 & 58,3 & 18 & 17,5 & 3 & 17,6 & 42 & 26,9 \\
\hline 25,0 a 29,9 & 13 & 36,1 & 48 & 46,6 & 6 & 35,3 & 67 & 43,0 \\
\hline 30,0 a 39,9 & 2 & 5,6 & 31 & 30,1 & 8 & 47,1 & 41 & 26,3 \\
\hline$>40,0$ & 0 & - & 6 & 5,8 & 0 & - & 6 & 3,8 \\
\hline Total & 36 & 100,0 & 103 & 100,0 & 17 & 100,0 & 156 & 100,0 \\
\hline \multicolumn{9}{|l|}{ RCQ } \\
\hline Homens $\leq 1,0$ & 10 & 83,3 & 21 & 63,6 & 2 & 33,3 & 33 & 64,7 \\
\hline$>1,0$ & 2 & 16,7 & 12 & 36,4 & 4 & 66,7 & 18 & 35,3 \\
\hline Total & 12 & 100,0 & 33 & 100,0 & 6 & 100,0 & 51 & 100,0 \\
\hline Mulheres $\leq 0,85$ & 16 & 66,7 & 32 & 45,7 & 3 & 27,3 & 51 & 48,6 \\
\hline$>0,85$ & 8 & 33,3 & 38 & 54,3 & 8 & 72,7 & 54 & 51,4 \\
\hline Total & 24 & 100,0 & 70 & 100,0 & 11 & 100,0 & 105 & 100,0 \\
\hline
\end{tabular}

* Nível A = PA normal (controlada); Nível B = HA leve a moderada; Nível C = HA severa + muito severa; $\bullet$ IMC = Índice de Massa Corporal; RCQ= Relação Cintura/Quadril. 
Foi detalhada a distribuição dos resultados segundo os pontos de corte do Índice de Massa Corporal (IMC) e da Relação Cintura/Quadril (RCQ), em cada nível de Pressão Arterial (PA) (Tabela 2). Na análise da amostra como um todo, pelo IMC, $73,1 \%$ dos pacientes apresentavam algum grau de excesso de peso, dos quais $26,3 \%$ e $3,8 \%$ foram classificados como portadores de obesidade moderada e grave, respectivamente. Quanto à RCQ, 35,3\% dos homens e $51,4 \%$ das mulheres mostraram valores típicos de obesidade andróide (RCQ > 1,0 e 0,85 , respectivamente) a qual, como foi visto anteriormente, possui maior valor preditivo de morbimortalidade que a quantidade total de gordura (Rimm et al., 1995; Lerário et al., 1997; Gillum et al., 1998; Rexrode et al., 1998).

É interessante ressaltar que o percentual de gordura no tronco superior (obesidade andróide), típica do sexo masculino, também foi extremamente freqüente, na amostra, entre as mulheres (51,4\% contra $35,3 \%$ dos homens). Apesar de esse diferencial por gênero não ter sido estatisticamente significante $\left(\chi^{2}=2,98 p=0,084\right)$, esse resultado demonstra que, além da perda da proteção dos estrógenos devido à idade, as mulheres neste estudo apresentavam um fator de risco adicional, a distribuição de gordura do tipo masculino.

A associação entre obesidade e hipertensão tem sido amplamente documentada pela literatura mundial (Gus et al., 1998; Kroke et al., 1998; Krummel, 1998; Calle et al., 1999). Tal fato foi evidenciado neste estudo (Tabela 2), pois apenas $5,6 \%$ dos indivíduos com PA controlada foram classificados como obesos moderados, não tendo sido registrado nenhum caso de obesidade severa, enquanto nos pacientes com HA leve e moderada essa freqüência ficou em torno de 30,1\% para obesidade moderada e 5,8\% para severa. Resultados similares foram encontrados entre os portadores de HA severa e muito severa, os quais, apesar de não terem manifestado níveis extremos de excesso de peso (IMC $\left.>40 \mathrm{~kg} / \mathrm{m}^{2}\right)$, apresentaram uma freqüência de obesidade moderada de quase $50 \%$.
Aplicando-se a análise estatística, verifica-se que o excesso de peso, de um modo geral, associou-se positivamente com o nível de $\mathrm{PA}$, informação comprovada pelo resultado do Qui-quadrado $\left(\chi^{2}=21,4 p=0,000\right)$.

Com a finalidade de avaliar, de forma isolada, o efeito dos diferentes níveis de excesso de peso, os dados foram reagrupados sob nova distribuição, reunindo em um grupo obesos leves mais os casos de normalidade, e em outro grupo os obesos moderados mais os casos classificados como grave. Com esse novo agrupamento, a análise mostrou haver diferença estatisticamente significante entre os indivíduos do primeiro grupo e os do segundo $\left(\chi^{2}=11,9\right.$ $p=0,000$ ). Entretanto, quando esse mesmo tipo de comparação foi realizada entre os normais e os pacientes com obesidade leve, os resultados também diferiram $\left(\chi^{2}=9,9 p=0,002\right)$, indicando que, neste estudo, qualquer grau de excesso de peso teve influência sobre os níveis de PA.

Contrariando tudo o que foi discutido acima sobre o padrão de distribuição de gordura (andróide ou ginecóide) e a freqüência de HA, neste trabalho não foi encontrada diferença estatisticamente significante entre os níveis de PA de homens (Fisher $p=0,113$ ) e mulheres $\left(\chi^{2}=3,19 p=0,074\right)$ classificados como "de risco" e "sem risco" pela RCQ.

Analisando a situação em termos de médias (Tabela 3), o IMC se situou dentro da faixa de obesidade leve tanto entre homens quanto entre mulheres $(\bar{X}=28,1 \pm 2,9$ DP e $\bar{X}=29,7 \pm 5,6$ $D P$, respectivamente), sendo ligeiramente superior no sexo feminino. Quanto aos valores encontrados para a RCQ, em ambos os sexos as médias se situaram bem próximas do valor limítrofe que determina a obesidade andróide e, portanto, a condição de risco, ou seja, $\bar{X}=0,97 \pm 0,12$ (homens) e $\bar{X}=0,84 \pm 0,09$ (mulheres).

Conforme indica a análise de variância (ANOVA) das médias acima, por nível de PA, nos pacientes estudados, independentemente do sexo, a PA não atuou como fonte de variação estatisticamente significante em nenhuma das duas variáveis analisadas. 
Tabela 3. Média $(\bar{X})$ e Desvio-Padrão (DP) do IMC e da RCQ • de hipertensos atendidos em $1{ }^{a}$ consulta no Ambulatório Geral de Nutrição do HC/UFPE, Recife, 1999.

\begin{tabular}{|c|c|c|c|c|c|c|c|c|c|}
\hline \multicolumn{10}{|c|}{ Níveis de Pressão Arterial (PA) } \\
\hline & \multicolumn{2}{|r|}{ *Nível A } & \multicolumn{2}{|r|}{ *Nível B } & \multicolumn{2}{|r|}{ *Nível C } & \multicolumn{2}{|r|}{ Total } & \multirow{2}{*}{ ANOVA } \\
\hline & $\mathrm{n}$ & $\bar{X} \pm D P$ & $\mathrm{n}$ & $\bar{X} \pm D P$ & $n$ & $\bar{X} \pm D P$ & $n$ & $\bar{x} \pm D P$ & \\
\hline \multicolumn{10}{|c|}{ IMC $\left(\mathrm{kg} / \mathrm{m}^{2}\right)$} \\
\hline Homens & 12 & $23,9 \pm 4,8$ & 33 & $28,7 \pm 2,4$ & 6 & $28,6 \pm 2,6$ & 51 & $28,1 \pm 2,9$ & {$[F(2,48)=2,88 \mathrm{NS}]$} \\
\hline Mulheres & 24 & $25,4 \pm 3,3$ & 70 & $29,4 \pm 5,9$ & 11 & $29,7 \pm 5,8$ & 105 & $29,7 \pm 5,6$ & {$[F(2,102)=1,12 \mathrm{NS}]$} \\
\hline \multicolumn{10}{|l|}{ RCQ } \\
\hline Homens & 12 & $0,79 \pm 0,01$ & 33 & $0,99 \pm 0,07$ & 6 & $1,09 \pm 0,12$ & 51 & $0,97 \pm 012$ & {$[\mathrm{~F}(2,48)=2,97 \mathrm{NS}]$} \\
\hline Mulheres & 24 & $0,78 \pm 0,02$ & 70 & $0,86 \pm 0,08$ & 11 & $0,89 \pm 0,09$ & 105 & $0,84 \pm 0,09$ & {$[F(2,102)=1,07 \mathrm{NS}]$} \\
\hline
\end{tabular}

* Nível A = PA normal (controlada); Nível B = HA leve a moderada; Nível C = HA severa + muito severa; $\bullet \mathrm{IMC}=$ Índice de Massa Corporal; RCQ= Relação Cintura/Quadril.

Tabela 4. Ingestão média de energia, macro e micronutrientes, colesterol, cloreto de sódio ( $\mathrm{NaCl}$ e e sódio de hipertensos atendidos no Ambulatório de Nutrição-HC/UFPE, Recife, 1999.

\begin{tabular}{lcccc}
\hline Nutrientes & Homens consumo & *RDA & Mulheres consumo & *RDA \\
\hline Energia (Kcal/dia) & $2125 \pm 403$ & $\cong 2500$ & $1540 \pm 600$ & $\cong 2000$ \\
Proteína (\% do VCT•) & 11,1 & 10 a 15 & 13,7 & 10 a 15 \\
Lipídeos (\% do VCT•) & 22,1 & 20 a 30 & 25,9 & 20 a 30 \\
Colesterol (mg/dia) & $207 \pm 97$ & $<300$ & $198 \pm 86$ & $<300$ \\
Cálcio (mg/dia) & $609 \pm 338$ & 800 & $753 \pm 429$ & 800 \\
Fibra (g/dia) & $21 \pm 8$ & 20 a 30 & $19 \pm 7$ & 20 a 30 \\
Potássio (mg/dia) & $2895 \pm 1046$ & $\cong 2000$ & $3024 \pm 1467$ & $\cong 2000$ \\
NaCl-sal de adição (g/dia) & $4,1 \pm 4$ & - & $4,0 \pm 4$ & - \\
Na* dietético total (mEq/dia) & 270 & 100 & 260 & 100 \\
\hline
\end{tabular}

* RDA = Recommended Dietary Allowances * Homem - 1,63m / 66 kg (Peso máximo aceitável); * Mulher - 1,51m / 57 kg (Peso máximo aceitável); * Atividade leve para ambos os sexos; * Na total = sódio do sal de adição + alimentos processados + alimentos in natura; $\bullet$ VCT $=$ Valor Calórico Total.

Com relação ao consumo de nutrientes da amostra (Tabela 4), convém ressaltar inicialmente que a ingestão média de energia referida pelos homens e mulheres pesquisados $(2125 \pm 403 \mathrm{kcal}$ e $1540 \pm 600 \mathrm{kcal}$, respectivamente) não justifica uma ocorrência tão elevada de excesso de peso, pois esses valores chegam a ser inferiores, principalmente entre as mulheres, aos considerados necessários para esses indivíduos se os mesmos apresentassem o peso máximo para a altura real $\left(I M C=24,9 \mathrm{~kg} / \mathrm{m}^{2}\right)$.

Entretanto, esses dados coincidem com os achados de Anjos et al. (1992), que tentaram explicar o crescente aumento da obesidade no Brasil, não pelo consumo energético, mas sim pela redução no gasto energético. De fato, analisando as alterações no perfil ocupacional (Oliveira et al., 1996), constata-se um crescimento significativo do setor terciário, o qual é composto basicamente por ocupações classificadas como "leves". Além disso, a modernização dos processos produtivos, inclusive na agricultura, também é responsável por essa acentuada redução na atividade física. No entanto, diante da complexidade do problema, outros fatores causais (além do consumo e gasto energético) certamente devem estar envolvidos.

No caso específico deste estudo, $77,6 \%$ dos indivíduos apresentaram atividade física ocupacional classificada como leve, o que é indicativo de uma população basicamente 
sedentária. Como a atividade física é um componente modificável do estilo de vida, mudanças nessa condição podem ser extremamente favoráveis em se tratando do paciente obeso.

Em relação à distribuição percentual dos nutrientes energéticos, o consumo de gordura esteve próximo do limite mínimo tido como aceitável $(20 \%)$, principalmente entre os homens, e o consumo de colesterol ficou abaixo do valor de $300 \mathrm{mg} /$ dia. Este resultado está de acordo com o estudo epidemiológico realizado por Bosch \& Pantin (1988), onde se evidenciou que o povo brasileiro apresenta um baixo consumo de gordura, quando comparado a outros países da América Latina.

Por outro lado, no tocante à proteína, os resultados são indicativos de uma ingesta dentro da normalidade para os homens e ligeiramente hiperprotéica para as mulheres. Salienta-se, a esse respeito, que a possível associação entre esse nutriente e a hipertensão arterial vem sendo bastante estudada nesses últimos anos. Entretanto, apesar de esses estudos sugerirem uma interrelação proteína/ pressão arterial, os resultados encontrados são ainda contraditórios, ou seja, alguns levantamentos epidemiológicos indicam não haver associação (Sacks \& Kass, 1988; Kestin et al., 1989) outros apontam uma relação inversa (Kihara et al., 1984; Reed et al., 1985), e há também uma pesquisa onde foi evidenciada uma elevação da PA com o aumento da proteína dietética (Sacks et al., 1981). Diante de tais controvérsias, Obarzanek et al. (1996), em uma revisão bibliográfica sobre o tema, observaram várias limitações metodológicas que poderiam explicar as discrepâncias acima, sugerindo a necessidade de estudos mais adequados e melhor controlados.

Em relação ao cálcio, existem evidências na literatura apontando para uma relação entre o seu baixo consumo e a ocorrência de hipertensão arterial (Campese \& Bianchi, 1997), confirmando os achados deste estudo, onde a ingestão ficou abaixo da Recommended Dietary Allowances (RDA) em ambos os sexos.

Outros dois nutrientes que também têm atraído a atenção dos pesquisadores são o potássio e a fibra. Com referência ao primeiro, muitos estudos epidemiológicos têm estabelecido uma relação inversa entre ele e a PA (Kaplan et al., 1985; Siani et al., 1987; Krishna \& Kapoor, 1991). A ação hipotensora do potássio pode ser decorrente de inúmeros mecanismos, como, por exemplo, sua ação de elevação da natriurese e diurese, além da diminuição da excreção urinária de cálcio e da secreção de renina e aldosterona (Campese \& Bianchi, 1997). Na amostra analisada, independentemente do sexo, o consumo mostrou-se adequado, com valores bem acima da Recommended Dietary Allowances de $2 \mathrm{~g} / \mathrm{dia}$.

Quanto à fibra, as especulações sobre sua capacidade de reduzir a PA não têm sido confirmadas pela literatura (Kestin et al., 1990; Swain et al., 1990). Entretanto, seu aumento na dieta pode implicar em uma alteração no conteúdo de lipídeos, o que certamente levaria a um padrão de alimentação bem mais saudável. Mesmo com essa indefinição sobre o verdadeiro papel da fibra no controle da PA, foi interessante constatar, na população em estudo, um consumo próximo (mulheres) ou dentro dos valores tidos como recomendados (homens), mostrando uma ingestão bem superior a média da população americana, que é de 11 a 13g/dia segundo Mahan \& Arlin (1995).

Por outro lado, apesar das limitações na metodologia utilizada para a verificação da ingestão de sal, pode-se sugerir um consumo elevado, com valores bem próximos (apenas para o sal adicionado aos alimentos), sem levar em conta o teor presente nos alimentos processados) de $6 \mathrm{~g} / \mathrm{dia}$, quantidade considerada recomendável para o hipertenso e, de certo modo, para a população em geral (Sixth Report..., 1997). De acordo com o estudo Intersalt, com 10079 homens e mulheres entre 20-59 anos de 32 países, uma 
diminuição na ingestão de sódio estava associada a uma redução na PA (Intersalt, 1988).

Contudo, deve-se salientar que o papel do sal na hipertensão permanece controverso. Tal controvérsia deriva em parte do reconhecimento de que seu efeito não é igual para todos os indivíduos, existindo os sensíveis e os não sensíveis ao sal, classificação baseada na resposta da PA a uma certa carga de cloreto de sódio. Na prática, devido à dificuldade de se fazer essa diferenciação, geralmente o sal é restrito para todos os hipertensos sem distinção (Krummel, 1998). Entretanto, a redução do sódio na dieta do hipertenso também está associada a outros benefícios, tais como: menor necessidade de drogas hipotensoras, diminuição da perda de potássio induzida por diuréticos, possível regressão da hipertrofia do ventrículo esquerdo e proteção contra a osteoporose e cálculos renais através da redução da calciúria (Sixth Report..., 1997). Desse modo, a restrição do sódio dietético é um fator a ser bem considerado. Neste estudo a ingestão total de sódio, incluindo o contido no sal de adição, nos alimentos processados e nos produtos in natura, ficou acima de $200 \mathrm{mEq}$, o que é característico de uma população com elevado teor dessa substância.

\section{O N C L U S Ã O}

Os dados deste estudo são sugestivos da influência do excesso de peso como fator de risco para a hipertensão, pois mais de $70 \%$ da amostra foi classificada dessa forma, tendo sido encontrada uma associação entre o excesso ponderal e o nível de pressão arterial. Quanto aos fatores dietéticos, destaca-se o elevado consumo de sódio, outro fator de risco ambiental, o qual, juntamente com o excesso de peso, "pode" e "deve" ser trabalhado em campanhas preventivas visando o controle desse mal.

Por outro lado, o consumo energético da amostra não se mostrou elevado, sugerindo que a falta de atividade física pode estar contribuindo para o excesso de peso e, conseqüentemente para a hipertensão. Contudo, a adoção de um estilo de vida mais saudável, com mudança de alguns hábitos alimentares e combate ao sedentarismo, não é algo inatingível e deve, portanto, ser estimulada por todo profissional de saúde envolvido com estratégias de prevenção primária da hipertensão arterial.

\section{REFERÊ NCIAS BIBLIOGRÁFICAS}

ACHUTTI, A.C., ACHUTTI, V.A.R. Aspectos epidemiológicos. In: AMODEO, C., LIMA, E.G., VAZQUEZ, E.C. Hipertensão arterial. São Paulo : Sarvier, 1997. p.11-21.

ADROGUÉ, H.J., WESSON, D.E. Role of dietary factors in the hypertension of African Americans. Seminars in Nefrology, Philadelphia, v.16, n.2, p.94-101, 1996

ALMEIDA, F. Hipertensão Arterial Essencial. In: RIELLA, M.C. Princípios de nefrologia e distúrbios hidroeletrolíticos. 3.ed. Rio de Janeiro : Guanabara Koogan, 1996. p.503-521.

ANJOS, L., SILVA, D.O., SERRÃO, S.A., SILVA, C.V.C. Vigilância nutricional em adultos: experiência de uma unidade de saúde atendendo a população favelada. Cadernos de Saúde Pública, Rio de Janeiro, v.8, n. 1, p.50-56, 1992.

BERTOLAMI, M.C. Hipertensão arterial e obesidade. Hiper Ativo, Rio de Janeiro, v.1, n.3, p. 44-56, 1994.

BOSCH, V.Y., PANTIN, E.L. Los grasos de las dietas. Archivos Latinoamericanos de Nutrición, Guatemala, v.38, n.3, p.506-518, 1988.

BURGOS, M.G.P.A., CABRAL, P.C., KOMURO, I., PIMENTEL, T. Perfil epidemiológico do atendimento ambulatorial de nutrição em um hospital universitário. In: CONGRESSO BRASILEIRO DE NUTRIÇÃO - SEGURANÇA ALIMENTAR E NUTRICIONAL NO BRASIL, 15., 1998, Belo Horizonte. Anais... Belo Horizonte : ASBRAN, 1998. p.81.

CABRAL, P.C. Homem, mulher e estado nutricional: um estudo em casais do Nordeste brasileiro, Recife, 
1994. 143p. Tese (Mestrado em Nutrição) - Centro de Ciências da Saúde, Universidade Federal de Pernambuco, 1994.

CALLE, E.E., THUN, M.J., PETRELLI, J.M., RODRIGUEZ, C., HEATH, C.W. Body mass index and mortality in a prospective cohort of U.S. adults. New England Journal of Medicine, Boston, v.341, n.15, p.1097-1105, 1999.

CAMPESE, V.M., BIANCHI, S. Nutritional factors in hypertension. In: KOPPLE, J.D., MASSRY, S.G. Nutritional management of renal disease. Baltimore : Williams \& Wilkins, 1997. p.77-95.

GILLUM, R.F., MUSSOLINO, M.E., MAOANS, J.H. Body fat distribution and hypertension incidence in women and men. The NHANES I epidemiologic follow-up study. International Journal of Obesity and Related Metabolic Disorders, Hampshire, v.22, n.2, p.127-134, 1998.

GUS, M., MOREIRA, L.B., PIMENTEL, M., GLEISENER, A.L.M., MORAES, R.S., FUCHS, F.D. Associação entre diferentes indicadores de obesidade e prevalência de hipertensão arterial. Arquivos Brasileiros de Cardiologia, São Paulo, v.70, n.2, p.111-114, 1998.

INTERSALT Cooperative Research Group: INTERSALT: an international study of electrolyte excretion and blood pressure. Results for 24 hours urinary sodium and potassium excretion. British Medical Journal, London, v.297, p.310-328, 1988.

KAPLAN, N.M., CARNEGIE, A., RASKIN, P., HELLER, J.A., SIMMONS, M. Potassium supplementation in hypertensive patients with diuretic-induced hypokalemia. New England Journal of Medicine, Boston, v.312, p.746-749, 1985.

KESTIN, M., ROUSE, I.L., CORREL, R.A., NESTEL, P.J. Cardiovascular disease risk factors in free-living men: comparison of two prudent diets, one based on lactoovovegetarianism and the other allowing lean meat. American Journal of Clinical Nutrition, Bethesda, v.50, n.2, p.280-287, 1989.

KESTIN, M., MOSS, R., CLIFTON, P.M., NESTEL, P.J. Comparative effects of three cereal brans on plasma lipids, blood pressure and glucose metabolism in mildly hypercholesterolemic men. American Journal of Clinical Nutrition, Bethesda, v.52, n.4, p.661-666, 1990.
KIHARA, M., FUJIKAWA, J., OHTAKA, M. Interrelationships between blood pressure, sodium, potassium, serum cholesterol and protein intake in Japanese. Hypertension, v.6, p.736-742, 1984.

KRISHNA, G.G., KAPOOR, S.C. Potassium depletion exacerbates essential hypertension. Annals of Internal Medicine, Lancaster, v.115, n.2, p.77-83, 1991.

KROKE, A., BERGMANN, M., KLIPSTEIN-GOBUSCH, K., BOEING, $\mathrm{H}$. Obesity body fat distribution and body build: their relation to blood pressure and prevalence of hypertension. International Journal of Obesity and Related Metabolic Disorders, Hampshire, v.22, n.11, p.1062-1070, 1998.

KRUMMEL, D. Nutrição na hipertensão. In: MAHAN, L.K., ARLIN, M.T. KRAUSE: alimentos, nutrição e dietoterapia. 9.ed. São Paulo : Roca, 1998. p.569-581.

LERÁRIO, A.C., BOSCO, A., ROCHA, M., SANTOMAURO, A.T., LUTHOID, W., GIANNELLA, D., WAJCHENBERG, B.L. Análise de fatores de risco em mulheres obesas e associação à gordura visceral. Diabetes \& Metabolism, São Paulo, v.1, p.39-45, 1997.

LESSA, I. Introdução à epidemiologia das doenças cardiovasculares no Brasil. In: LESSA, I. O adulto brasileiro e as doenças da modernidade: epidemiologia das doenças crônicas não-transmissíveis. São Paulo : Hucitec, 1998. p.43-72.

MAHAN, L.K., ARLIN, M.T. Carboidratos. In: MAHAN, L.K., ARLIN, M.T. KRAUSE: alimentos, nutrição e dietoterapia. 8.ed. São Paulo : Roca, 1995. p.29-44.

MASSIE, B.M. Systemic Hypertension. In: Current: medical diagnosis \& treatment. Stamford: Appleton \& Lange, 1997. p.412-431.

MUST, A., JACQUES, P.F., DALLAL, G.E., BAJEMA, C.J., DIETZ, W.H. Long term morbidity and mortality of overweight adolescents. New England Journal of Medicine, Boston, v.5, n. 19, p.1350-1354, 1992.

NATIONAL RESEARCH COUNCIL (USA). Recommended Dietary Allowances. 10.ed. Washington DC: National Academy of Science, 1989. 284p. (Food and Nutrition Board). 
NATIONAL CHOLESTEROL EDUCATION PROGRAM EXPERT PANEL. Adult Treatment Panel II: Summary of the Second Report of the National Cholesterol Education Program Expert Panel on detection, evaluation, and treatment of high blood cholesterol in adults. Journal of the American Medical Association, Chicago, v.269, n.23, p.3015-3023, 1993.

OBARZANEK, E., VELLETRI, P.A., CUTLER, J.A. Dietary protein and blood pressure. Journal of the American Medical Association, Chicago, v.275, n.20, p.22-29, 1996.

OLIVEIRA, J.E.D., CUNHA, S.F.C., MARCHINI, J.S. A desnutrição dos pobres e dos ricos: dados sobre a alimentação no Brasil. São Paulo : Sarvier, 1996. p.17-18.

PINOTTI, J.A. Por um sistema de atenção integral à mulher. Revista do Instituto Materno Infantil de Pernambuco, Recife, v.1, n. 1, p.103-115, 1987.

PROGRAMA DE APOIO À NUTRIÇÃO. Versão 2.5. Centro de Informática em Saúde da Escola Paulista de Medicina. São Paulo, 1993 (Software).

REED, D., McGEE, D., YANO, K., HANKIN, J. Diet, blood pressure, and multicollonearity. Hypertension, v.7, p.405-410, 1985.

REXRODE, K.M., CAREY, V.J., HENNEKENS, C.H., WALTERS, E.E., COLDITZ, G.A., STAMPFER, M.J., WILLET, W.C., MANSON, J.E. Abdominal adiposity and coronary heart disease in women. Journal of the American Medical Association, Chicago, v. 280, n.21, p.1843-1848, 1998.

RIMM, E.B., STAMPFER, M.J., GIOVANNUCCI, E., ASCHERIO, A., SPIEGELMAN, D., COLDITZ, G.A., WILLETT, W.C. Body size and fat distribution as predictors of coronary heart disease among middle-aged and older US men. American Journal Epidemiology, Baltimore, v.141, n.12, p.1117-1127, 1995.

SACKS, F.M., DONNER, A., CASTELLI, W.P., GRONEMEYER, J., PLETKA, P., MARGOLIUS, H.S., LANDSBERG, L., KASS, E.H. Effect of ingestion of meat on plasma cholesterol of vegetarians. Journal of the American Medical Association, Chicago, v.246, n.6, p.640-644, 1981.

SACKS, F.M., KASS, E.H. Low blood pressure in vegetarians: effects of specific foods and nutrients.
American Journal of Clinical Nutrition, Bethesda, v.48, n.3, p.795-800, 1988.

SIANI, A., STRAZZULLO, P., RUSSO, L., GUGLIELMI, S., IACOVIELLO, L., FERRARA, L.A., MANCINI, M. Controlled trial of long-term oral potassium supplements in patients with mild hypertension. British Medical Journal, London, v.294, n.6585, p.1453-1456, 1987.

SILVEIRA, C.A.M., REMÍGIO, M.I., BRANDÃO, S. Hipertensão Arterial Sistêmica. In: FIGUEIRA, N.M., COSTA JÚNIOR, J.I., SÁ LEITÃO, C.C., LUCENA, V.G., MELO, H.R.L., BRITO, C.A.A. Condutas em clínica médica. 2.ed. Rio de Janeiro : Medsi, 2001. p.314-332.

SIXTH Report of the Joint National Committee on Prevention, Detection, Evaluation, and Treatment of High Blood Pressure (The). The Joint National Committee on Detection, Evaluation, and Treatment of High Blood Pressure. Archives Internal Medicine, Chicago, v.157, p.2413-2446, 1997.

SWAIN, J., ROUSE, I.L., CURLEY, C.B., SACKS, F.M. Comparison of the effects of oat bran and low-fiber wheat on serum lipoprotein levels and blood pressure. New England Journal of Medicine, Boston, v.322, p.147-154, 1990.

VERAS, R.P., RAMOS, L.R., KALACHE, A. Crescimento da população idosa no Brasil: transformações e conseqüências na sociedade. Revista de Saúde Pública, São Paulo, v.21, n.3, p.225-233, 1987.

WILLIAMS, P.T., FORTMANN, S.P., TERRY, R.B. Association of dietary fat, regional adiposity and blood pressure in men. Journal of the American Medical Association, Chicago, v.257, n.23, p.3251-3256, 1987.

WORLD HEALTH ORGANIZATION. Expert Committee: physical status: the use and interpretation of anthropometry. Geneva, 1995. (Technical Report Series, n. 854).

WORLD HEALTH ORGANIZATION. Epi Info. Version 6.04b. A word processing, database and statistic program for public health. [programa de computador]. Genebra, 1997.

Recebido para publicação em 20 de março de 2000 e aceito em 13 de dezembro de 2001. 